\title{
Retrospective investigation of postoperative mid-term results of cryoablation and radiofrequency ablation methods used in atrial fibrillation surgery treatment
}

\author{
Ahmet Burak Tatlı ${ }^{1}$, Arda Aybars Pala ${ }^{2} \oplus$, Mesut Engin ${ }^{3} \oplus$, Temmuz Taner $^{4} \oplus$, Yusuf Ata $^{3} \odot$ \\ ${ }^{1}$ Department of Cardiovascular Surgery, Bursa City Hospital, Bursa, Turkey \\ ${ }^{2}$ Department of Cardiovascular Surgery, Adlyaman Training and Research Hospital, Adryaman, Turkey \\ ${ }^{3}$ Department of Cardiovascular Surgery, University of Health Sciences, Bursa Yüksek İhtisas Training and Research Hospital, Bursa, \\ Turkey \\ ${ }^{4}$ Department of Cardiovascular Surgery, Mardin State Hospital, Mardin, Turkey
}

\section{ABSTRACT}

Objectives: Atrial fibrillation (AF) is the most common arrhythmia encountered and is usually seen in patients scheduled for coronary artery bypass and mitral valve surgery. Radiofrequency ablation and cryoablation are two methods used in AF surgery and proven efficacy. In this study, cryoablation and radiofrequency ablation methods were compared in terms of clinical outcomes, efficacy and safety.

Methods: Between November 2011 and September 2017; 99 patients with AF who underwent radiofrequency ablation or cryoablation during open heart surgery were included in this study with 2 groups. The patients who underwent cryoablation were defined as Group I $(n=40)$, and the patients who underwent radiofrequency ablation as Group II $(n=59)$. Preoperative, perioperative, early and mid (1 year) postoperative period characteristics of the groups were analyzed.

Results: The mean age was $60.6 \pm 9$ years in Group I and $60.7 \pm 9.1$ years in Group II $(p=0.960)$. When the operative values were examined, ablation time and cross-clamp time was found to be low in Group II and a statistically significant difference was found ( $p<0.001$ and $p=0.043$; respectively). When the rhythms of the postoperative first year controls are examined, sinus rhythm was observed in 34 (85\%) patients in Group I and $50(84.7 \%)$ patients in Group II. There was no statistically significant difference in return to sinus rhythm in the first year $(p=0.975)$.

Conclusions: The success rates of these two methods used in AF surgery are effective but they are not superior to each other.

Keywords: Atrial fibrillation, cryoablation, radiofrequency ablation, surgical ablation

A trial fibrillation (AF) is a common arrhythmia in the society. It was first described in 1909 by Thomas Lewis. While the incidence in the general population is between $0.4 \%$ and $2 \%$, this rate reaches $10 \%$ over the age of 60 . This rate was reported to be between $30 \%$ and $84 \%$ in patients scheduled for surgery for mitral valve, and $5 \%$ in patients scheduled for surgery for coronary heart disease [1].

$\mathrm{AF}$ has been perceived as a benign arrhythmia for many years; therefore, treatment options have been

Received: November 5, 2020; Accepted: December 8, 2020; Published Online: April 20, 2021

How to cite this article: Tatl BT, Pala AA, Engin B, Taner T, Ata Y. Retrospective investigation of postoperative mid-term results of cryoablation and radiofrequency ablation methods used in atrial fibrillation surgery treatment. Eur Res J 2021;7(5):501-508. DOI: 10.18621/eurj.814878

Address for correspondence: Arda Aybars Pala, MD., Adlyaman Training and Research Hospital, Department of Cardiovascular Surgery, Yunusemre Mah., 1164 Sk., No: 13, 02200 Merkez, Adlyaman, Turkey.E-mail: ardaaybars@hotmail.com, Mobil: +90 532 7104587, Fax: +904162145399 
limited. However, several previous studies showed that $\mathrm{AF}$ increases the probability of stroke by six times, doubling the mortality due to cardiovascular causes. Even in the absence of stroke, it was shown that AF probably causes cognitive impairment as a result of silent lacuner and cortical infarctions or hypoperfusion. Persistent AF was associated with doubled mortality due to all causes and cardiovascular disease-related [2-4].

Approaches in surgical treatment targeted to stop uncontrolled electrical triggers in the atrium and to ensure atrial contraction function. For this purpose, left atrial isolation procedure, catheter ablation of sense node, corridor procedure, pulmonary button isolation, atrial compartment operations were applied, respectively throughout the history. Finally, the surgery that was called "Maze III Procedure", which was developed by Cox and which was modified twice, became the gold standard for AF treatment with $99 \%$ success. Today, radiofrequency (RF) ablation and cryoablation methods are used widely in the surgical treatment of AF [5]. Success of AF treatment with isolated left atrial RF ablation and cryoablation has been reported to be $70-85 \%$ [6-8].

The purpose of this study was to determine the efficacy and reliability, and early and mid-term clinical results after RF ablation and cryoablation methods used for AF therapy in patients who have AF rhythm in the preoperative period and have undergone open heart surgery.

\section{METHODS}

A total of 99 patients at the University of Health Sciences, Bursa Yuksek Ihtisas Training and Research Hospital, Bursa, Turkey, between November 2011 and September 2017, who underwent open heart surgery with cardiopulmonary bypass (CPB), who had AF in the preoperative period, and who underwent RF ablation or cryoablation during the surgery were included in the study in 2 groups (Group I: Cryoablation, Group II: RF ablation). The study was designed retrospectively based on the database, and was approved with the decision of Uludag University Faculty of Medicine Clinical Research Ethics Committee on 21.11.2017 with the number 2017-17/3.

The preoperative, perioperative, early and mid (1 year) postoperative period features of patients were recorded. Patients with endocarditis, sick sinus syndrome, calcification in the left atrium wall, who did not admit to our hospital for routine outpatient followups after discharge, and who did not complete their first 1-year follow-ups were excluded from the study. All patients were examined with transthoracic echocardiography (TTE) in the preoperative period. In the echocardiography unit of our hospital, left atrium (LA) diameter and ejection fraction (EF) were calculated using the Teichholz formula from two-dimensional parasternal long axis images with the G.E Electronics Vivid 7 Echocardiography device. The pulmonary artery pressure (PAP) was measured with continuous wave (CW) method through the tricuspid valve in patients who had tricuspid insufficiency. In patients who did not have tricuspid insufficiency, it was measured with pulmonary flow acceleration time.

\section{Surgical Technique}

Aorta-bicaval cannulation was performed after median sternotomy, and CPB was started after the ACT (activated clotting time) was $>400$. After crossclamping, if there was coronary bypass grafting $(\mathrm{CABG})$ in the surgical procedure to be performed, distal anastomosis of saphena grafts was performed firstly. If the surgery to be performed was valve surgery, left atriotomy was performed.

After left atriotomy, firstly, left atrium and appendix were evaluated. The first thing to do was thrombectomy in cases with thrombus in the left atrial appendix, and the left atrial appendix was ligated internally with $4 / 0$ prolene suture. If valve replacement was planned, the mitral valve was excised (if possible, posterior leaflet was preserved). The right pulmonary veins were circled starting from the incision made from the interatrial groove, then the left pulmonary veins were circled in one single circle to form a set of lesions. Then another set of lesions was created to combine the two candidates that included the right and left pulmonary veins from the superior segment. This line was positioned towards the left atrium ceiling as much as possible to avoid possible esophageal damage. Another ablation line was created to combine the left pulmonary veins and the mitral valve posterior annulus after the ablation line extending from the left atrial appendix to the left superior pulmonary vein was created. Another line that started exactly in the middle 
of this line was extended towards the atrium base to prevent "re-entry" waves that might occur between atriums through the coronary sinus.

As the precaution for this, ablation was performed before additional surgical procedure in order not to damage the suture lines. In addition, wet gauze was placed in the LA posterior before the ablation to prevent possible esophageal damage, and the transesophageal echocardiography (TEE) probe was withdrawn (if any). The ablation line was directed towards P2-P3 to avoid damage to the circumflex artery during the ablation process. The Cardioblate CryoFlex Surgical Ablation Probe (Medtronic Inc., Minneapolis, MN, USA) was used in Group I, and the Irrigated Unipolar Cardioblate ${ }^{\circledR}$ Surgical Ablation Pen was used in Group II (Medtronic Inc., Minneapolis, MN, USA).

The concomitant surgery procedure was performed after the ablation procedure was completed. The cross-clamp was removed after the surgical procedure was completed, and CPB was terminated. Temporary epicardial "pacemaker" wires were placed in patients at the end of the surgery against AV block risk.

\section{Postoperative Medical Treatment and Follow-up}

In our clinic, our medical treatment protocol for patients who undergo surgical ablation is administering intravenous Amiodaron (1200 mg) in the first 24 hours postoperatively, to every patient without discrimination. Half of this treatment dose $(600 \mathrm{mg})$ is given in the form of loading dose after the crossclamping, and the rest is given in the form of maintenance dose. Amiodaron oral tablet is initiated (600 $\mathrm{mg}$ /day), and is used as $200 \mathrm{mg}$ /day for at least 3 months after the discharge.

Amiodaron infusion was started without discrimination in the groups of patients included in our study at the loading dose (600 mg) after cross-clamping, and maintenance Amiodaron infusion $(600 \mathrm{mg}$ ) was initiated after the loading dose. Following decannulation, internal cardioversion was applied to patients who had AF. Patients were taken to the intensive care unit and followed up with full monitoring. Rhythm follow-up was carried out with 12-derivation electrocardiography (ECG). In the intensive care unit, patients were re-evaluated in terms of rhythm, and patients without hemodynamic problems were taken to the ward. Patients were discharged with planned anticoagulant and antiarrhythmic treatment. The ECG data and postop- erative $6^{\text {th }}$ month TTE follow-up results were recorded in routine follow-ups of patients after the discharge (3rd month, 6th month, and 1st year).

\section{Statistical Analysis}

The SPSS 21.0. (IBM Corp. Armonk, NY: USA. Released 2012) program was used to analyze the data obtained in the study, and $p<0.05$ was taken statistically significant. While analyzing the study data, besides descriptive statistical methods (Mean \pm standard deviation); Student t-test or Mann-Whitney U-test was used in cross-group comparisons of parameters that showed normal distribution or not in the comparison of the quantitative data; Chi-Square Test was used to compare the qualitative data.

\section{RESULTS}

There were 40 patients in Group I, and Group II included 59 patients in our study. Isolated left atrial ablation was applied to all patients. The mean age of the patients was $60.6 \pm 9$ years in Group I; and the mean age of the patients in Group II was $60.7 \pm 9.1(p$ $=0.960)$. Among the patients in Group I, $26(65 \%)$ were females, and 14 (35\%) were males; and in Group II, 35 (59.4\%) were female, and 24 (40.6\%) were male $(p=0.569)$. When the preoperative patient characteristics were evaluated, no statistically significant differences were detected between the groups in terms of chronic obstructive pulmonary disease, diabetes mellitus, hypertension, and coronary artery disease. The demographic characteristics of patients are presented in Table 1.

When the groups were compared in terms of preoperative EF, EF values of patients in Group II were lower than patients in Group I and statistically significant differences were found (Group I: $54 \pm 7.5$ vs Group II: $50.25 \pm 6.1, p=0.014)$. There were no statistically significant differences between the other TTE parameters that were evaluated; preoperative LA diameter and PAP values ( $p=0.327$ and $p=0.947$; respectively) (Table 1).

Considering the surgical procedures applied to the patients; Mitral valve replacement in $48(48.5 \%)$ patients, mitral valve replacement with aortic valve replacement in $13(13.1 \%)$ patients, tricuspid ring annuloplasty with mitral valve replacement in 12 
Table 1. Demographic and preoperative features of the patients

\begin{tabular}{lccc}
\hline Variables & Group I $(\mathbf{n}=\mathbf{4 0})$ & Group II $(\mathbf{n}=\mathbf{5 9})$ & $\boldsymbol{p}$ value \\
\hline Age (years) & $60.6 \pm 9(40-80)$ & $60.7 \pm 9.1(36-82)$ & 0.960 \\
Gender (Male/Female) & $26 / 14$ & $35 / 24$ & 0.569 \\
COPD & $10(25 \%)$ & $15(25.4 \%)$ & 0.962 \\
DM & $7(17.5 \%)$ & $13(22 \%)$ & 0.581 \\
HT & $13(32.5 \%)$ & $14(23.7 \%)$ & 0.336 \\
CAD & $7(17.5 \%)$ & $10(16.9 \%)$ & 0.943 \\
EF $(\%)$ & $54 \pm 7.5(40-65)$ & $50.25 \pm 6.1(35-65)$ & $\mathbf{0 . 0 1 4}$ \\
PAP (mmHg) & $48.1 \pm 9.3(30-70)$ & $48.2 \pm 8.4(30-70)$ & 0.947 \\
LA diameter $(\mathrm{mm})$ & $56.2 \pm 6.7(43-70)$ & $55.1 \pm 6.8(44-77)$ & 0.327 \\
\hline
\end{tabular}

Data are shown as mean \pm standard deviation (minimum-maximum) or number (\%). COPD $=$ Chronic obstructive pulmonary disease, $\mathrm{DM}=$ Diabetes mellitus, $\mathrm{HT}=$ Hypertension, $\mathrm{CAD}=$ Coronary artery disease, $\mathrm{EF}=\mathrm{Ejection}$ fraction, $\mathrm{PAP}=$ Pulmonary artery pressure, $\mathrm{LA}=$ Left atrium

\section{Table 2. Surgical procedures}

\begin{tabular}{lccc}
\hline Procedure & Group I $(\mathbf{n}=\mathbf{4 0})$ & Group II (n= 59) & Total (n=99) \\
\hline AVR + MVR & $5(12.5 \%)$ & $8(13.5 \%)$ & $13(13.1 \%)$ \\
MVR & $20(50 \%)$ & $28(47.4 \%)$ & $48(48.5 \%)$ \\
MRA & $4(10 \%)$ & $7(11.8 \%)$ & $11(11.1 \%)$ \\
MVR + CABG & $3(7.5 \%)$ & $3(5.1 \%)$ & $6(6.1 \%)$ \\
MRA + CABG & $1(2.5 \%)$ & $2(3.4 \%)$ & $3(3 \%)$ \\
AVR + MRA & 0 & $2(3.4 \%)$ & $2(2 \%)$ \\
AVR + MVR + TRA & $1(2.5 \%)$ & $1(1.7 \%)$ & $2(2 \%)$ \\
MVR + TRA & $6(15 \%)$ & $6(10.2 \%)$ & $12(12.1 \%)$ \\
MVR + TRA + CABG & 0 & $1(1.7 \%)$ & $1(1 \%)$ \\
MRA + TRA & 0 & $1(1.7 \%)$ & $1(1 \%)$ \\
\hline
\end{tabular}

Data are shown as number (\%). AVR $=$ Aortic valve replacement, $\mathrm{MVR}=$ Mitral valve replacement, MRA = Mitral ring annuloplasty, $\mathrm{CABG}=$ Coronary artery bypass grafting, $\mathrm{TRA}=$ Tricuspid ring annuloplasty

$(12.1 \%)$ patients and mitral ring annuloplasty in 11 $(11.1 \%)$ patients. In addition, CABG was applied as an additional surgical procedure in $10(10.1 \%)$ patients. Surgical procedures applied to patients are shown in Table 2.

Both groups were compared in terms of perioperative total perfusion times (TPt), cross-clamp times $(\mathrm{CCt})$ and ablation times. Mean TPt was $113.5 \pm 11$ minutes in Group I, 109.6 \pm 10.1 minutes in Group II; mean CCt was 76.8 \pm 7.3 minutes in Group I and 74.1 \pm 10.4 minutes in Group II. While no statistically significant difference was found between the groups in terms of TPt, CCt was significantly lower in Group II ( $p=0.101$ and $p=0.043$; respectively). When the mean ablation times were examined, it was found that it was $13.7 \pm 1$ minutes in Group I and $8.6 \pm 1$ minutes in Group II, and this difference was statistically significant $(p<0.001)$ (Table 3$)$.

When the operation output rhythms of the patients were evaluated, Sinus rhythm was achieved at a rate of $80 \%$ in Group I and $79.7 \%$ in Group II, and no significant difference was found $(p=0.967)$. In routine follow-ups of the groups after the discharge, 1 patient in Group I and 2 patients in Group II returned to AF rhythm during the follow-ups in the 3rd month. During the follow-ups in the $6^{\text {th }}$ month, it was seen that 3 patients in Group I and 5 patients in Group II returned to sinus rhythm. In statistical terms, no statistical differences were detected between the groups compared to the $3^{\text {rd }}$ and 6th month sinus rhythm rates $(p=0.961$ 
Table 3. Perioperative data

\begin{tabular}{lccc}
\hline Variables & Group I $(\mathbf{n}=\mathbf{4 0})$ & Group II $(\mathbf{n}=\mathbf{5 9})$ & $\boldsymbol{p}$ value \\
\hline $\mathrm{TPt}(\mathrm{min})$ & $113.5 \pm 11(95-141)$ & $109.6 \pm 10.1(96-150)$ & 0.101 \\
$\mathrm{CCt}(\mathrm{min})$ & $76.8 \pm 7.3(68-111)$ & $74.1 \pm 10.4(58-115)$ & $\mathbf{0 . 0 4 3}$ \\
Ablation time $(\mathrm{min})$ & $13.7 \pm 1(12-15)$ & $8.6 \pm 1(7-10)$ & $<\mathbf{0 . 0 0 1}$ \\
\hline
\end{tabular}

Data are shown as mean \pm standard deviation (minimum-maximum). $\mathrm{TPt}=$ Total perfusion time, $\mathrm{CCt}=\mathrm{Cross}$-clamp time

Table 4. Early and mid-term rhythm results of the patients

\begin{tabular}{lccc}
\hline Variables & Group I $(\mathbf{n}=\mathbf{4 0})$ & Group II $(\mathbf{n}=\mathbf{5 9})$ & $\boldsymbol{p}$ value \\
\hline Postoperative sinus rhythm & $32(80 \%)$ & $47(79.7 \%)$ & 0.967 \\
$3^{\text {rd }}$ month sinus rhythm & $31(77.5 \%)$ & $45(76.2 \%)$ & 0.961 \\
$6^{\text {th }}$ month sinus rhythm & $34(85 \%)$ & $50(84.7 \%)$ & 0.975 \\
$1^{\text {st }}$ year sinus rhythm & $34(85 \%)$ & $50(84.7 \%)$ & 0.975 \\
\hline
\end{tabular}

Data are shown as number $(\%)$.

Table 5. Sixth month transthoracic echocardiography data of the patients

\begin{tabular}{lllc}
\hline Variables & Group I $(\mathbf{n}=\mathbf{4 0})$ & Group II $(\mathbf{n}=\mathbf{5 9})$ & $\boldsymbol{p}$ value \\
\hline EF $(\%)$ & $53.1 \pm 6.9(35-65)$ & $49.5 \pm 6.7(30-60)$ & $\mathbf{0 . 0 1 3}$ \\
PAP $(\mathrm{mmHg})$ & $36.7 \pm 7.3(25-50)$ & $37.5 \pm 6.3(25-55)$ & 0.633 \\
LA diameter $(\mathrm{mm})$ & $47.3 \pm 4.6(39-56)$ & $46.1 \pm 4.8(38-61)$ & 0.117 \\
\hline
\end{tabular}

Data are shown as mean \pm standard deviation (minimum-maximum). EF $=$ Ejection fraction, PAP $=$ Pulmonary artery pressure, $\mathrm{LA}=$ Left atrium

and $p=0.975$; respectively). In the 1 st year of followups, it was determined that sinus rhythm continued at a rate of $85 \%$ in Group I and $84.7 \%$ in Group II, and no statistically significant difference was found $(p=$ 0.975). In line with the data obtained, no return to sinus rhythm was observed after the $6^{\text {th }}$ month followups (Table 4).

When the 6th month TTE results were evaluated, it was seen that there were statistically weakly significant differences between the groups compared in EF values $(p=0.013)$; and there were similar values with EF values in the preoperative period. In the 6th month, no statistically significant differences were detected between the groups in terms of LA diameter and PAP values ( $p=0.633$ and $p=0.117$, respectively) (Table $5)$.

\section{DISCUSSION}

AF increases mortality and morbidity at significant levels, as well as significantly impairs the quality of life of patients and imposes a significant socioeconomic burden on patients. Arrhythmia is the cause of approximately $10 \%$ of hospitalizations related to the circulatory system. Most of this is composed of AF and atrial flutter $[3,6]$. In patients who undergo cardiac surgery, the achieving sinus rhythm in the postoperative period is very important for healing process $[9,10]$.

The groups in our study were those who underwent irrigated unipolar RF ablation and cryoablation, and all of them had pathology in the mitral valve. In the literature, the presence of AF rhythm was reported in $30-79 \%$ of patients who undergo valve surgery and the rate of spontaneous return to postoperative sinus rhythm was less than $10 \%[11,12]$. However, Forlani et al.'s [13] study argued that the return to sinus rhythm increased the survival rates of patients and reduces morbidity significantly. For this purpose, the application of ablation therapy together with mitral intervention will be beneficial in patients with persistent AF rhythm in the preoperative period.

Several complications might develop due to this 
process. The complications reported so far in the literature consist of endocardial applications. Regardless of the probe used, bleeding due to esophageal damage or left atrium perforation may develop especially in left atrial RF ablation. Phrenic nerve damage may occur during cryoablation. However, cryoablation has low thrombogenicity compared with RF ablation, with a low perioperative bleeding and atrial wall perforation risk [14]. The most fatal complication in endocardial practice is esophageal damage [15]. During the circling of the right and left pulmonary vein mouths in two separate islets and the combination of these two, the esophageal may be damaged due to its neighboring localization to left atrium posterior wall. In our clinical applications, to avoid such complications and minimize the possibility of damage, the TEE probe and nasogastric catheter are removed (if any) during the ablation process, and gauze is placed in the oblique sinus. Also, attention must be paid not to intersect the left atrial circles because applying energy to the same tissue for a second time increases the risk of perforation [16]. Doll et al. [15] applied RF ablation to 387 patients, and reported that esophageal perforation developed in four patients (1\%). Also, circumflex artery injury during Maze Operation combined with cryoablation, left main bronchial injury because of its proximity to the posterior wall of the left atrium, and pulmonary vein stenosis can also be named among related complications $[17,18]$. No complications were detected in our patients in our study, including esophageal damage and left atrial wall perforation.

In the present study, it was observed that TPt was shorter in patients who underwent RF ablation compared to those who underwent cryoablation. In his study, Güden et al. [19] reported that the endocardial procedure lasted between 9 and 12 minutes for left atrial ablation. In our study, cryoablation lasted 12-15 minutes, and RF ablation process lasted 7-10 minutes. It was observed in our study that RF ablation process was significantly shorter, when the ablation application times were compared between both groups ( $p<$ 0.001). When TPt and CCt, which included ablation and surgical procedure, were compared, no statistically significant difference was found between the two groups in terms of TPt. In terms of CCt, CCt was found to be significantly shorter in the RF ablation group $(p=0.043)$.

The amputation of atrial appendices, especially the left atrial appendix, is still controversial today. However, with the risk of thromboembolic events, it is still considered as a logical approach to perform atrial amputation, or at least applying ablation to the base, then sewing it from the inside, or by inserting a pouch suture from the outside, closing the mouth completely [20]. We also think in line with this approach in our clinic, and apply internal ligation to the left atrial appendix in the framework of our surgical ablation protocol.

One important point that stands out in all studies reporting RF ablation results was that although the rates of recovering from $\mathrm{AF}$ after surgery were always close to $100 \%$, a significant decrease also occurred, especially in the first week, followed by a re-increase in six months to one year. In our study, it was found that there was a return from sinus rhythm to $\mathrm{AF}$ rhythm in the first 3 months ( 1 patient in Group I, and 2 patients in Group II). After 1st year follow-up, it was found that 3 patients in cryoablation group and 5 patients in RF ablation group returned to sinus rhythm in the last nine months. This condition is explained with temporary shortening of the refractory period of atrium because of edema, inflammation and high catecholamine levels in circulation as a result of surgical trauma in the postoperative period [21]. However, AF can also be seen in early postoperative period because of advanced age, increased sympathetic activity, ischemia and electrolyte disorder, atrial factors, and other underlying cardiac diseases. This period lasts about 3 months, and patients are advised to continue their antiarrhythmic treatments. For this reason, Geidel et al. [22] recommended using "Amiodaron" in the first 3 months. Antiarrhythmic treatment (Amiodaron) is applied to all patients in the first 3 months of postoperative period according to the medical treatment protocol of our clinic after cryoablation and radio-frequency ablation. For this reason, six-month, even one year and above follow-up times should be considered in the evaluation of surgical treatment results of AF. In terms of clinical applications, it must also be noted that AF attacks in early postoperative period may be temporary. It should be known that the actual result will be determined in a longer period.

There are numerous studies in the literature that compare cryoablation and RF ablation methods in terms of clinical outcomes, efficacy and safety. In the case control study of Linhart et al. [23] with 40 pa- 
tients with paroxysmal AF, they found that cryoablation and RF ablation had similar success rates. Kojodjojo et al. [24] compared cryoablation and RF ablation in 124 patients with persistent and paroxysmal AF. The success rate at the end of one year follow-up was reported to be $77 \%$ in cryoablation group, and $72 \%$ in RF ablation group. Kühne et al. [25] conducted a study and compared cryoablation and RF ablation procedures, $88 \%$ success rate was reported in cryoablation group, and $92 \%$ success rate in RF ablation group in one year follow-up. In the "Fire and Ice" study published by Kuck et al. [26], 762 patients who underwent cryoablation and RF ablation were followed-up in two groups for approximately 18 months, and had no significant differences when the effectiveness and reliability of the two methods were compared. Also, a large-scale meta-analysis study compiled by Hachem et al. [27] consisting of 247 randomized controlled trials found similar results with "Fire and Ice" study. In our study, at the end of the 1 st year, $85 \%$ success rate was reported in cryoablation group, and $84.7 \%$ in RF ablation group with no statistically significant differences between the groups $(p=0.975)$. The success rates we achieved in our study showed similar results with these studies in terms of the comparison of cryoablation and RF ablation methods, and suggested that both methods are not superior to each other, and their efficacy is similar.

\section{Limitations}

The fact that the number of patients was low in our study, its retrospective design, high diversity of patients, and lack of a long follow-up period were the limitations of our study. In this respect, we believe that large-scale studies should be conducted with larger patient series and longer follow-up periods.

\section{CONCLUSION}

As a conclusion, sinus rhythm was achieved at a high rate with cryoablation and irrigated unipolar RF ablation applied to patients in preoperative AF rhythm scheduled for open heart surgery. When they were compared in terms of postoperative morbidity, mortality, rhythm control and echocardiographic values, it was observed that both methods were effective and reliable; however, at the same time they had no superi- ority over each other.

\section{Authors' Contribution}

Study Conception: ABT; Study Design: ABT, AAP; Supervision: YA; Funding: ABT; Materials: ABT, AAP, ME; Data Collection and/or Processing: ABT, ME, TT; Statistical Analysis and/or Data Interpretation: AAP, YA; Literature Review: ABT, ME, TT; Manuscript Preparation: ABT, AAP, TT and Critical Review: YA.

\section{Conflict of interest}

The authors disclosed no conflict of interest during the preparation or publication of this manuscript.

\section{Financing}

The authors disclosed that they did not receive any grant during the conduction or writing of this study.

\section{REFERENCES}

1. Hunter RJ, Baker V, Finlay MC, Duncan ER, Lovell MJ, Tayebjee MH, et al. Point-by-point radiofrequency ablation versus the cryoballoon or a novel combined approach: a randomized trial comparing 3 methods of pulmonary vein isolation for paroxysmal atrial fibrillation (The Cryo versus RF Trial). J Cardiovasc Electrophysiol 2015;26:1307-14.

2. Erdolu B, As AK. The relationship between postoperative atrial fibrillation after coronary artery bypass surgery and serum heat shock protein levels and inflammation. Eur Res J 2020;6:409-15. 3. Pala AA, Urcun YS. Is the mean platelet volume a predictive factor for atrial fibrillation developing after coronary artery bypass grafting in elderly patients? Heart Surg Forum 2020;23:E809-14.

4. Kirchhof P, Benussi S, Kotecha D, Ahlsson A, Atar D, Casadei B, et al. 2016 ESC Guidelines for the management of atrial fibrillation developed in collaboration with EACTS. Eur J Cardiothorac Surg 2016;50:e1-e88.

5. Melby SJ, Schuessler RB, Damiano RJ Jr. Ablation technology for the surgical treatment of atrial fibrillation. ASAIO J 2013;59:461-8.

6. Barnett SD, Ad N. Surgical ablation as treatment for the elimination of atrial fibrillation: a meta-analysis. J Thorac Cardiovasc Surg 2006;131:1029-35.

7. Chavez EK, Colafranceschi AS, Monteiro AJO, Canale LS, Mesquita ET, Weksler C, et al. Surgical treatment of atrial fibrillation in patients with rheumatic valve disease. Braz J Cardiovasc Surg 2017;32:202-209.

8. McCarthy PM, Manjunath A, Kruse J, Andrei AC, Li Z, McGee EC Jr, et al. Should paroxysmal atrial fibrillation be treated during cardiac surgery? J Thorac Cardiovasc Surg 2013;146:810-23. 
9. Güvenç O, Göncü MT, Engin M, Çayır MÇ, Özyazıcıŏlu AF. Effects of coronary endarterectomy on postoperative early results in long segment coronary artery disease. Eur Res J 2020;6:18792.

10. Öztürk C, Yavuz Ş. Effect of coronary artery bypass surgery on ventricular functions in patients with poor left ventricular function. Eur Res J 2019;5:502-9.

11. Yang S, Mei B, Feng K, Lin W, Chen G, Liang M, et al. LongTerm Results of Surgical Atrial Fibrillation Radiofrequency Ablation: Comparison of Two Methods. Heart Lung Circ 2018;27:621-8.

12. Jessurun ER, van Hemel NM, Kelder JC, Elbers S, de la Rivière $A B$, Defauw $J J$, et al. Mitral valve surgery and atrial fibrillation: is atrial fibrillation surgery also needed? Eur J Cardiothorac Surg 2000;17:530-7.

13. Forlani S, De Paulis R, Guerrieri Wolf L, Greco R, Polisca P, Moscarelli M, et al. Conversion to sinus rhythm by ablation improves quality of life in patients submitted to mitral valve surgery. Ann Thorac Surg 2006;81:863-7.

14. Mugnai G, Chierchia GB, de Asmundis C, Sieira-Moret J, Conte G, Capulzini L, et al. Comparison of pulmonary vein isolation using cryoballoon versus conventional radiofrequency for paroxysmal atrial fibrillation. Am J Cardiol 2014;113:1509-13.

15. Doll N, Borger MA, Fabricius A, Stephan S, Gummert J, Mohr FW, et al. Esophageal perforation during left atrial radiofrequency ablation: Is the risk too high? J Thorac Cardiovasc Surg 2003; 125:836-42.

16. Gillinov AM, Pettersson G, Rice TW. Esophageal injury during radiofrequency ablation for atrial fibrillation. J Thorac Cardiovasc Surg 2001;122:1239-40.

17. Sueda T, Shikata H, Mitsui N, Nagata H, Matsuura Y. Myocardial infarction after a maze procedure for idiopathic atrial fibrillation. J Thorac Cardiovasc Surg 1996;112:549-50.

18. Benussi S, Nascimbene S, Calvi S, Alfieri O. A tailored anatomical approach to prevent complications during left atrial ablation. Ann Thorac Surg 2003;75:1979-81.

19. Güden M, Akpinar B, Sanisoğlu I, Sağbaş E, Bayindir O. In- traoperative saline-irrigated radiofrequency modified Maze procedure for atrial fibrillation. Ann Thorac Surg 2002;74:S1301-6. 20. Ramlawi B, Abu Saleh WK, Edgerton J. The left atrial appendage: target for stroke reduction in atrial fibrillation. Methodist Debakey Cardiovasc J 2015;11:100-3.

21. Knecht S, Sticherling C, von Felten S, Conen D, Schaer B, Ammann P, et al. Long-term comparison of cryoballoon and radiofrequency ablation of paroxysmal atrial fibrillation: a propensity score matched analysis. Int J Cardiol 2014;176:645-50.

22. Geidel S, Ostermeyer J, Lass M, Betzold M, Duong A, Jensen $\mathrm{F}$, et al. Three years experience with monopolar and bipolar radiofrequency ablation surgery in patients with permanent atrial fibrillation. Eur J Cardiothorac Surg 2005;27:243-9.

23. Linhart M, Bellmann B, Mittmann-Braun E, Schrickel JW, Bitzen A, Andrié R, et al. Comparison of cryoballoon and radiofrequency ablation of pulmonary veins in 40 patients with paroxysmal atrial fibrillation: a case-control study. J Cardiovasc Electrophysiol 2009;20:1343-8.

24. Kojodjojo P, O'Neill MD, Lim PB, Malcolm-Lawes L, Whinnett ZI, Salukhe TV, et al. Pulmonary venous isolation by antral ablation with a large cryoballoon for treatment of paroxysmal and persistent atrial fibrillation: medium-term outcomes and non-randomised comparison with pulmonary venous isolation by radiofrequency ablation. Heart 2010;96:1379-84.

25. Kühne M, Suter Y, Altmann D, Ammann P, Schaer B, Osswald $\mathrm{S}$, et al. Cryoballoon versus radiofrequency catheter ablation of paroxysmal atrial fibrillation: biomarkers of myocardial injury, recurrence rates, and pulmonary vein reconnection patterns. Heart Rhythm 2010;7:1770-6.

26. Kuck KH, Brugada J, Fürnkranz A, Metzner A, Ouyang F, Chun KR, et al. Cryoballoon or radiofrequency ablation for paroxysmal atrial fibrillation. N Engl J Med 2016;374:2235-45. 27. Hachem AH, Marine JE, Tahboub HA, Kamdar S, Kanjwal $\mathrm{S}$, Soni R, et al. Radiofrequency ablation versus cryoablation in the treatment of paroxysmal atrial Fibrillation: a meta-analysis. Cardiol Res Pract 2018;2018:6276241. 